ANNALES

POLONICI MATHEMATICI

$80(2003)$

\title{
On approximation by special analytic polyhedral pairs
}

\author{
by V. ZAhariuta (Istanbul and Ankara) \\ Dedicated to Professor Józef Siciak \\ on the occasion of his seventieth birthday
}

\begin{abstract}
For bounded logarithmically convex Reinhardt pairs "compact set - domain" $(K, D)$ we solve positively the problem on simultaneous approximation of such a pair by a pair of special analytic polyhedra, generated by the same polynomial mapping $f: D \rightarrow \mathbb{C}^{n}, n=\operatorname{dim} \Omega$. This problem is closely connected with the problem of approximation of the pluripotential $\omega(D, K ; z)$ by pluripotentials with a finite set of isolated logarithmic singularities $([23,24])$. The latter problem has been solved recently for arbitrary pluriregular pairs "compact set - domain" $(K, D)$ by Poletsky [12] and S. Nivoche $[10,11]$, while the first one is still open in the general case.
\end{abstract}

1. Introduction. The problem of approximation of the Green pluripotential $\omega(D, K ; z)$ by pluripotentials with a finite set of isolated logarithmic singularities $([23,24])$, which is of great importance in Complex Potential Theory, has been solved recently (E. Poletsky [12], S. Nivoche [10, 11]) for general pluriregular pairs $(K, D)$; in the particular case of Reinhardt pairs it was proved independently in [25] in a different way (see Corollary 4 below). The above problem is closely connected with the problem of simultaneous approximation of a pluriregular pair "open set - compact set" $D \supset K$ by a pair of special analytic polyhedra, generated by the same mapping $f: D \rightarrow \mathbb{C}^{n}$ (see Problem 2 below). In the one-dimensional case both problems were studied long ago: moreover, approximation of the Green potential by partial sums of its integral representation (as well as approximation of a pair $(D, K)$ by a pair of lemniscates) is a powerful tool in analytic function theory (see, e.g., $[20,13,19]$ ).

In the present paper, which is a revised version of the preprint [25] updated in connection with the above mentioned results of Poletsky and Nivoche, we give a positive answer to the second problem for Reinhardt

2000 Mathematics Subject Classification: 32A07, 32U20.

Key words and phrases: pluripotential, Reinhardt domains, special analytic polyhedron. 
pairs in $\mathbb{C}^{n}$. This result is based on simultaneous reduction of the frames of analytic polyhedral pairs under certain quite special conditions, which hold for similar $n$-circular polyhedral pairs (Lemma 2). The construction used for that reduction is of independent interest: for example, it was applied, after some generalization, in [2] to strengthen the classical Lelong-Bremermann Lemma (see, e.g., [5, Q]) by proving that the number $N$ of analytic fuctions involved is bounded by $2 n+1$.

It is quite obvious that a positive answer to the second problem yields automatically a positive solution of the first one, while the converse conclusion, as far as I know, is still open in the general case.

We also consider some applications in approximation theory and discuss the connection of the above problems with an extremal problem in Complex Analysis (cf. [24, item 3.2.5]).

Acknowledgements. The author would like to express his gratitude to Professor A. Aytuna for fruitful discussions and useful remarks, and to the referee for a considerable improvement of the manuscript.

2. Preliminaries. Let $D$ be a bounded pseudoconvex domain on a Stein manifold $\Omega$ and $K$ be a compact subset of $D$. The Green pluripotential $\omega(D, K ; z)$ of $K$ with respect to $D$ was introduced by J. Siciak [16] (see also $[17,18,22,15])$ :

$$
\omega(z)=\omega(D, K ; z)=\varlimsup_{\zeta \rightarrow z} \sup \left\{u(\zeta): u \in P(D), u \leq 1,\left.u\right|_{K} \leq 0\right\},
$$

where $P(D)$ stands for the set of all plurisubharmonic functions in $D$.

The pair $(K, D)$ is said to be pluriregular if $\omega(z) \leq 0$ on $K$ and $\omega\left(z_{k}\right) \rightarrow 1$ for any sequence $\left\{z_{k}\right\}$ having no limit points in $D$; everywhere in this paper, when using the term "pluriregular pair" we will assume that the following two additional natural conditions are fulfilled: (a) $A(D)$ is dense in $A(K)$ (this is some sort of Runge condition); (b) $D$ has no components disjoint from $K$ (which is a dual Runge condition, impying that $A(K)^{*}$ is densely embedded into $\left.A(D)^{*}\right)$.

The complex Monge-Ampère operator $\left(d d^{c}\right)^{n}$ (see [3]) is defined for any bounded plurisubharmonic function $u$ in $D$ so that $\left(d d^{c} u\right)^{n}$ is a non-negative Borel measure on $D$ (here $d=\partial+\bar{\partial}, d^{c}=i(\bar{\partial}-\partial)$ ). In particular, for a pluriregular pair we get a CPT analogue of the equilibrium measure $\mu_{0}(K, \Omega):=\left(d d^{c} \omega\right)^{n}$, supported by $K$ (see [3]).

The pluricapacity $\tau(K, \Omega)$ of $K$ with respect to $\Omega$ (or of the condenser $(K, \Omega))$ is the number

$$
\tau(K, \Omega)=(2 \pi)^{-n} \int_{K}\left(d d^{c} \omega(\Omega, K ; z)\right)^{n},
$$


which differs from the Bedford-Taylor pluricapacity ([3]) only by a constant factor.

The multipolar Green pluripotential for an open set $D \subset \Omega$ with a given sequence $\Lambda=\left(\lambda_{1}, \ldots, \lambda_{m}\right) \in D^{m}$ of logarithmic poles and distribution of measures $\alpha=\left(\alpha_{1}, \ldots, \alpha_{m}\right) \in \mathbb{R}_{+}^{n}$ is the following extremal plurisubharmonic function:

$$
g_{D}(\Lambda, \alpha ; z):=\limsup _{\zeta \rightarrow z} \sup \{u(\zeta): u \in G(D, \Lambda, \alpha)\}
$$

where $G(D, \Lambda, \alpha)$ is the set of all functions $u \in P(D)$ such that $u \leq 0$ in $D$ and $u(\zeta) \leq \alpha \ln \left|\zeta-\lambda_{j}\right|+$ const in a neighborhood of any point $\lambda_{j}$, $j=1, \ldots, m$.

We will use the notation $D_{s} \Uparrow D$ to mean that $D_{s}$ and $D$ are open sets, $D_{s}$ is relatively compact in $D_{s+1}, s \in \mathbb{N}$, and $D=\bigcup_{s=1}^{\infty} D_{s} ; K_{s} \Downarrow K$ means that $K_{s}$ and $K$ are compact sets, $K_{s+1} \subset \operatorname{int} K_{s}$, and $K=\bigcap_{s=1}^{\infty} K_{s}$.

3. Two problems. We discuss two important problems of Complex Potential Theory.

Problem $1([23,24])$. Let $(K, D)$ be a pluriregular pair. Do there exist $\Lambda^{(s)} \in K^{m_{s}}$ and $\alpha^{(s)} \in \mathbb{R}_{+}^{m_{s}}, m_{s} \in \mathbb{N}, s \in \mathbb{N}$, such that

$$
g_{D}\left(\Lambda^{(s)}, \alpha^{(s)} ; z\right) \rightarrow \omega(D, K ; z)-1
$$

uniformly on any compact subset of $\bar{D} \backslash K$ ?

For the one-dimensonal case this problem has an immediate positive answer: it is sufficient to take an appropriate sequence of integral sums of the integral representing the Green potential. The lack of such representation in the multidimensional case was for a long time a serious obstacle to attacking Problem 1, which has been solved only recently in $[12,10,11]$ (the particular case of Reinhardt pairs was done independently, in a different way, in [25], see also Corollary 4 below).

Notice that the solution of this problem itself may be considered as an efficient substitution for an integral representation, especially in connection with many applications.

Let $D$ be an open set on a Stein manifold $\Omega, \operatorname{dim} \Omega=n$, and $f: D \rightarrow \mathbb{C}^{m}$ be an analytic mapping, $m \geq n$. We say that $(L, G)$ is a similar analytic polyhedral pair in $D$ (represented by the mapping $f$ ) if there exists an open subset $U$ in $D$ and multiradii $\mathbf{r}^{(\nu)}=\left(r_{j}^{(\nu)}\right) \in \mathbb{R}_{+}^{m}, \nu=0,1, r_{j}^{(0)}<r_{j}^{(1)}$, $j=1, \ldots, m$, such that

$$
\begin{aligned}
& G=\left\{z \in U:\left|f_{j}(z)\right|<r_{j}^{(1)}, j=1, \ldots, m\right\}, \\
& L=\left\{z \in G:\left|f_{j}(z)\right| \leq r_{j}^{(0)}, j=1, \ldots, m\right\},
\end{aligned}
$$


and $G$ is relatively compact in $U$. The quadruple $\left[U, f ; \mathbf{r}^{(0)}, \mathbf{r}^{(1)}\right]$ is called the frame of the pair $(L, G)$; to stress that the polyhedral pair is generated by $m$ analytic functions we will speak about $m$-polyhedral pairs or $m$-frames. If $m=n$ we say that the polyhedral pair $(L, G)$ is special; a special polyhedral pair is a natural multidimensional analogue of a lemniscate pair in $\mathbb{C}$, generated by a single analytic function.

The following statement is known as the Lelong-Bremermann Lemma $([9,4]$, see also $[5, \mathrm{Q}])$ :

Proposition 1. Let $u$ be a continuous plurisubharmonic function on a pseudoconvex domain $D$. Then for each compact subset $A$ of $D$ and any $\varepsilon>0$ there exists an analytic mapping $f=\left(f_{j}\right): D \rightarrow \mathbb{C}^{N}$ and numbers $\alpha_{j}>0$ such that

$$
\left|\omega(z)-\max \left\{\alpha_{j} \ln \left|f_{j}(z)\right|: j=1, \ldots, N\right\}\right|<\varepsilon, \quad z \in A .
$$

A drawback of this result is that, in general, the number $N=N(\varepsilon, A)$ in (6) may increase without bound.

It is proved in [2], using the reduction suggested in the next section, that the constant $N$ has a bound $\leq 2 n+1, n=\operatorname{dim} D$. Since the plurisubharmonic function $\omega(z)=\omega(D, K ; z)$ is continuous in $D$ for any pluriregular pair $(K, D)$ (see [22]), one can derive from this that for any pluriregular pair $(K, D)$ there exists a sequence of similar analytic polyhedral pairs $\left(L_{s}, G_{s}\right)$ generated by analytic mappings $f^{(s)}: D \rightarrow \mathbb{C}^{2 n+1}$ such that $G_{s} \Uparrow D$ and $L_{s} \Downarrow K$. But it is well known that in the one-dimensional case any regular pair $(K, L)$ can be approximated by pairs of analytic polyhedra (lemniscates) generated by single analytic functions $f_{s}: D_{s} \rightarrow \mathbb{C}$, with $D_{s} \uparrow D$ (see, e.g., $[20,8.7]$; this is a natural development of Hilbert's result [6] about approximating a simple Jordan curve by polynomial lemniscates). So, for the multidimensional case the following problem arises naturally:

Problem 2. Is it possible to approximate any pluriregular pair $(K, D)$ simultaneously by special similar analytic polyhedral pairs?

This problem can be reformulated in the following equivalent form.

Problem 2a. For any pluriregular pair $(K, D), \operatorname{dim} D=n$, find a sequence of analytic mappings $f^{(s)}=\left(f_{j}^{(s)}\right): D \rightarrow \mathbb{C}^{n}$ and vectors $\alpha^{(s)}=$ $\left(\alpha_{j}^{(s)}\right) \in \mathbb{R}_{+}^{n}$ such that the sequence

$$
u^{(s)}(z):=\max \left\{\alpha_{j}^{(s)} \ln \left|f_{j}^{(s)}\right|: j=1, \ldots, n\right\}
$$

converges to $\omega(D, K ; z)-1$ uniformly on any compact subset of $D \backslash K$.

In the present paper we solve this problem positively for Reinhardt pairs $(K, D)$. 
Notice that an approach to general pluriregular pairs $(K, D)$ is given in $[10,11]$ (see also [12]). Namely, it is proved there that such a pair can be approximated by pairs (5) if in the expression for $L$ the set $G$ is replaced by some open set $V \supset K$. So, in that context, instead of the uniform convergence on compact subsets of $D \backslash K$ for the sequence (7), a weaker condition is proved: $\int_{D}\left(d d^{c}\left(u^{(s)}(z)-\omega(z)\right)\right)^{n} \rightarrow 0$ as $s \rightarrow \infty$.

4. Reduction of analytic polyhedral pairs. Any similar analytic $m$-polyhedral pair with a frame $\left[D, f ; \mathbf{r}^{(0)}, \mathbf{r}^{(1)}\right]$ can be represented by its normalized frame:

$$
[D, g ; \mathbf{r}]:=[D, g ; \mathbf{1}, \mathbf{r}],
$$

where $g=\left(f_{j} / r_{j}^{(0)}\right), \mathbf{1}:=(1, \ldots, 1), \mathbf{r}:=\left(r_{j}^{(1)} / r_{j}^{(0)}\right)$.

We say that an analytic polyhedral pair with the frame (8) is equilateral if

$$
\mathbf{r}=r \cdot \mathbf{1}, \quad r \in \mathbb{R}_{+} .
$$

Let $(L, G)$ be an equilateral similar analytic polyhedral pair with the $m$ frame (8), (9). For any $J=\left\{j_{1}, \ldots, j_{k}\right\}$ with $j_{1}<\ldots<j_{k}, 1 \leq k \leq m$, we define

$$
\sigma(J):=\left\{z \in \overline{G \backslash L}:\left|g_{j_{1}}(z)\right|=\ldots=\left|g_{j_{k}}(z)\right|>\left|g_{j}(z)\right|, j \notin J\right\}
$$

Suppose that there exists $l$ with $n \leq l<m$ such that

$$
\sigma(J)=\emptyset
$$

if $\# J>l$, and $l$ is the smallest number satisfying this condition. Then we consider the sequence of analytic mappings

$$
\begin{aligned}
g^{(s)} & =\left(g_{1}^{(s)}, \ldots, g_{l}^{(s)}\right): D \rightarrow \mathbb{C}^{l}, \\
g_{k}^{(s)}(z) & =\left(\sum_{j_{1}<\ldots<j_{k}}\left(g_{j_{1}}(z)\right)^{s} \ldots\left(g_{j_{k}}(z)\right)^{s}\right)^{l ! / k},
\end{aligned}
$$

where $k=1, \ldots, l$.

Lemma 2. Let $(L, G)$ be an equilateral polyhedral pair with the frame (8) such that the condition (10) holds. Let

$$
\begin{aligned}
\varphi(z): & =\frac{1}{\ln r} \max \left\{\ln \left|g_{j}(z)\right|: j=1, \ldots, m\right\}, \\
\varphi_{s}(z): & =\frac{1}{s l ! \ln r} \max \left\{\ln \left|g_{k}^{(s)}(z)\right|: k=1, \ldots, l\right\},
\end{aligned}
$$

where $g_{k}^{(s)}(z)$ are defined in $(11)$. Then $\varphi_{s}(z) \rightarrow \varphi(z)$ uniformly on $\overline{G \backslash L}$.

Proof. Since, by the construction,

$$
\left|g_{k}^{(s)}(z)\right| \leq 2^{m l ! / k}\left(\max \left\{\left|g_{j}(z)\right|: j=1, \ldots, m\right\}\right)^{s l !},
$$


we get an estimate from above:

$$
\varphi_{s}(z) \leq \varphi(z)+\frac{m \ln 2}{s k \ln r}
$$

for each $z \in \overline{G \backslash L}$.

Now we deal with the estimate from below for the function (13). First, by the continuity of $\varphi$ on $\overline{G \backslash L}$, for each $\varepsilon>0$ we can find $\sigma(\varepsilon)>0$ such that

$$
|\varphi(z)-\varphi(\zeta)|<\varepsilon \quad \text { if }|\zeta-z|<\sigma(\varepsilon), z, \zeta \in \overline{G \backslash L} .
$$

Now we fix any $\zeta \in \overline{G \backslash L}$. By the hypothesis, there is $J=J(\zeta)=\left\{j_{1}, \ldots, j_{k}\right\}$, $k=k(\zeta) \leq l$, such that

$$
\left|g_{j_{1}}(\zeta)\right|=\ldots=\left|g_{j_{k}}(\zeta)\right|>\left|g_{i}(\zeta)\right|, \quad i \notin J
$$

hence

$$
d(\zeta):=\max \left\{\left|\frac{g_{i_{1}}(\zeta) \ldots g_{i_{k}}(\zeta)}{g_{j_{1}}(\zeta) \ldots g_{j_{k}}(\zeta)}\right|: I=\left\{i_{1}, \ldots, i_{k}\right\} \neq J\right\}<1
$$

Thus, using the continuity of all the functions involved, we can find a neighborhood $U(\zeta)=\{z \in \overline{G \backslash L}:|z-\zeta|<\varepsilon\}$ with $\delta(\zeta)<\sigma(\varepsilon)$ such that

$$
\left|\frac{g_{i_{1}}(z) \ldots g_{i_{k}}(z)}{g_{j_{1}}(z) \ldots g_{j_{k}}(z)}\right| \leq q(\zeta):=\frac{1+d(\zeta)}{2}<1, \quad I=\left\{i_{1}, \ldots, i_{k}\right\} \neq J
$$

and

$$
\left|g_{j}(z)\right| \geq r^{-\varepsilon}\left|g_{j}(\zeta)\right|, \quad j \in J
$$

for all $z \in U(\zeta)$. Applying (11), (18), and (19) we obtain the estimate

$$
\begin{aligned}
\left|g_{k}^{(s)}(z)\right| & \geq\left|g_{j_{1}}(z) \ldots g_{j_{k}}(z)\right|^{s l ! / k}\left(1-\sum_{I \neq J}\left|\frac{g_{i_{1}}(z) \ldots g_{i_{k}}(z)}{g_{j_{1}}(z) \ldots g_{j_{k}}(z)}\right|^{s}\right)^{l ! / k} \\
& \geq r^{-\varepsilon s l !}\left|g_{j_{1}}(\zeta) \ldots g_{j_{k}}(\zeta)\right|^{s l ! / k}\left(1-2^{m} q(\zeta)^{s}\right)^{l ! / k}
\end{aligned}
$$

for every $z \in U(\zeta)$. Hence taking into account (12), (13), and (15), we deduce that

$$
\begin{aligned}
\varphi_{s}(z) & \geq \frac{\ln \left|g_{k}^{(s)}(z)\right|}{s l ! \ln r} \geq \frac{\ln \left|g_{j_{1}}(\zeta)\right|}{\ln r}+\frac{\ln \left(1-2^{m} q(\zeta)\right)}{k \ln r}-\varepsilon \\
& \geq \varphi(\zeta)-2 \varepsilon \geq \varphi(z)-3 \varepsilon
\end{aligned}
$$

for $z \in U(\zeta)$ and $s \geq s_{0}(\zeta, \varepsilon)$.

Now, choosing a finite covering: $\overline{G \backslash L} \subset \bigcup_{i=1}^{N} U\left(\zeta^{(i)}\right)$, we conclude that

$$
\varphi^{(s)}(z) \geq \varphi(z)-3 \varepsilon
$$

for all $z \in \overline{G \backslash L}$ if $s \geq s_{0}(\varepsilon):=\max \left\{s_{0}\left(\zeta^{(i)}, \varepsilon\right): i=1, \ldots, N\right\}$.

The estimates (14) and (20) imply that $\varphi_{s}(z)$ converges to $\varphi(z)$ uniformly on $\overline{G \backslash L}$. 
5. Pluripotentials for Reinhardt pairs. Let $D$ be a logarithmically convex bounded complete $n$-circular domain in $\mathbb{C}^{n}$. Its characteristic function

$$
h_{D}(\theta)=\sup \left\{\sum \theta_{k} \ln \left|z_{k}\right|: z \in D\right\}, \quad \theta \in \overline{\mathbb{R}_{+}^{n}},
$$

is convex and homogeneous. The domain $D$ can be recovered from its characteristic function as follows:

$$
D=\left\{z \in \mathbb{C}^{n}: \sum \theta_{k} \ln \left|z_{k}\right|<h_{D}(\theta), \theta \in \Sigma\right\},
$$

where $\Sigma:=\left\{\theta=\left(\theta_{1}, \ldots, \theta_{n}\right) \in \overline{\mathbb{R}_{+}^{n}}: \sum_{k=1}^{n} \theta_{k}=1\right\}$.

Let $D_{0}, D_{1}$ be a pair of bounded logarithmically convex complete Reinhardt (=n-circular) domains such that $\bar{D}_{0} \subset D_{1}$. The following formula for the pluripotential of $\bar{D}_{0}$ with respect to $D_{1}$ was presented in [23, Proposition 1.4.3]:

$$
\omega(z)=\omega\left(D_{1}, \bar{D}_{0} ; z\right)=\sup \{\gamma(\theta, z): \theta \in \Sigma\}
$$

for $z \in \bar{D}_{1} \backslash D_{0}$, where

$$
\gamma(\theta, z):=\frac{\sum_{\nu=1}^{n} \theta_{\nu} \ln \left|z_{\nu}\right|-h_{D_{0}}(\theta)}{h_{D_{1}}(\theta)-h_{D_{0}}(\theta)} .
$$

The formula (21) is extended onto $\partial D_{1}$ by setting $\omega(z) \equiv 1$ there.

We consider the following level sets of the function $\omega$ :

$$
D_{\alpha}:=\left\{z \in D_{1}: \omega(z)<\alpha\right\}, \quad \Gamma_{\alpha}:=\left\{z \in \bar{D}_{1}: \omega(z)=\alpha\right\}
$$

with $\alpha \in[0,1]$.

It is easy to see that the representation (21) leads to the following geometric description of the level sets (23).

Lemma 3. Let $0<\alpha<1$. Then $\Gamma_{\alpha}$ is the boundary of the domain $D_{\alpha}$ and $z=\left(z_{\nu}\right) \in D_{1}$ belongs to $\Gamma_{\alpha}$ if and only if there exist $\theta \in \Sigma$, $z^{(0)}=\left(z_{\nu}^{(0)}\right) \in \Gamma_{0}, z^{(1)}=\left(z_{\nu}^{(1)}\right) \in \Gamma_{1}$ such that $\left|z_{\nu}\right|=\left|z_{\nu}^{(0)}\right|^{1-\alpha} \cdot\left|z_{\nu}^{(1)}\right|^{\alpha}$ and $\gamma\left(\theta, z^{(0)}\right)=0, \gamma\left(\theta, z^{(1)}\right)=1$.

Using these facts and Lemma 2 we are going to prove our main result, which gives a positive solution of Problem 2 in the case considered.

Theorem 4. Let $D_{0}, D_{1}$, and $\omega(z)$ be as above. Then there exist a sequence of polynomial mappings $f^{(\nu)}=\left(f_{j}^{(\nu)}\right): \mathbb{C}^{n} \rightarrow \mathbb{C}^{n}$, a sequence $\alpha_{\nu}>0$, and a sequence of open sets $G^{(\nu)} \Uparrow D_{1}$ such that

$$
\omega(z)=\lim _{\nu \rightarrow \infty} \alpha_{\nu} \max \left\{\ln \left|f_{j}^{(\nu)}(z)\right|: j=1, \ldots, n\right\}
$$

uniformly on any compact subset of $D_{1} \backslash \bar{D}_{0}$, and a sequence of special polynomial polyhedral pairs $\left(M^{(\nu)}, H^{(\nu)}\right)$ determined by the normalized equilateral 
frames

$$
\left[G^{(\nu)}, f^{(\nu)} ; \exp 1 / \alpha_{\nu}\right]
$$

approximates the pair $\left(\bar{D}_{0}, D_{1}\right)$ so that $H_{\nu} \Uparrow D_{1}$ and $M_{\nu} \Downarrow \bar{D}_{0}$.

As a corollary we get another proof of Problem 1 in the case considered (cf. $[12,10,11])$.

Corollary 5. Let $K=\bar{D}_{0}, D=D_{1}$. Then in the setting of the previous theorem, for each $\nu$ the set $\Lambda^{(\nu)}:=\left\{\zeta \in G^{(\nu)}: f^{(\nu)}(\zeta)=0\right\}$ is finite and consists only of simple roots, and the relation $(4)$, with $\alpha^{(\nu)}:=\left(\alpha_{\nu}, \ldots, \alpha_{\nu}\right) \in$ $\mathbb{R}^{n}$, holds uniformly on any compact subset of $D \backslash K$.

Before proving these statements we consider the following

Lemma 6. Let $D_{0}, D_{1}$, and $\omega$ be as above. Then for each $\varepsilon>0$ there exists a finite set of multi-indices $k(j)=\left(k_{i}(j)\right) \in \mathbb{Z}_{+}^{n}$, a natural number $q$, and real numbers $c_{j}, j=1, \ldots, m$, such that the maximum

$$
v(z):=\frac{1}{q} \max \left\{\sum_{i=1}^{n} k_{i}(j) \ln \left|z_{i}\right|-c_{j}: j=1, \ldots, m\right\}
$$

is attained for no more than $n$ values of $j$ at any point $z$ satisfying the estimates $0 \leq v(z) \leq 1$, and

$$
|\omega(z)-v(z)|<\varepsilon, \quad z \in \overline{D_{1} \backslash D_{0}} .
$$

Proof. First we notice that for each $\zeta=\left(\zeta_{i}\right) \in \overline{D_{1} \backslash D_{0}}$ there is $\theta=\theta(\zeta)$ such that

$$
\omega(\zeta)=\gamma(\theta(\zeta), \zeta)
$$

Indeed, denoting by $\Sigma(\zeta)$ the set of all $\theta=\left(\theta_{i}\right) \in \Sigma$ such that $\theta_{i}=0$ whenever $\zeta_{i}=0$, we have

$$
\omega(\zeta)=\sup \{\gamma(\theta, \zeta): \theta \in \Sigma(\zeta)\},
$$

and the function $\gamma(\theta, \zeta)$ is continuous in $\theta$ on the compact set $\Sigma(\zeta)$, so (28) is valid with some $\theta=\theta(\zeta) \in \Sigma(\zeta)$.

Now, since $\omega$ is continuous on $\bar{D}_{1}$, while the function $\gamma(\theta(\zeta), z)$ is continuous in some neighborhood of $\zeta$, we can find for any $\varepsilon>0$ some open neighborhood $U_{\zeta}$ of $\zeta$ such that

$$
0 \leq \omega(z)-\gamma(\theta(\zeta), z)<\varepsilon / 2, \quad z \in U_{\zeta}
$$

Hence, using the covering theorem, we deduce that for each $\varepsilon>0$ there is a finite set $\left\{\zeta^{(j)}: j=1, \ldots, m\right\}$ such that

$$
|\omega(z)-u(z)|<\varepsilon / 2, \quad z \in \overline{D_{1} \backslash D_{0}},
$$


where

$$
u(z):=\sup \left\{\sum_{i=1}^{m} a_{i, j} \ln \left|z_{i}\right|-b_{j}: j=1, \ldots, m\right\}
$$

and

$$
\begin{aligned}
a_{i, j} & :=\frac{\theta\left(\zeta^{(j)}\right)}{h_{D_{1}}\left(\theta\left(\zeta^{(j)}\right)\right)-h_{D_{0}}\left(\theta\left(\zeta^{(j)}\right)\right)}, \\
b_{j} & :=\frac{h_{D_{0}}\left(\theta\left(\zeta^{(j)}\right)\right)}{h_{D_{1}}\left(\theta\left(\zeta^{(j)}\right)\right)-h_{D_{0}}\left(\theta\left(\zeta^{(j)}\right)\right)} .
\end{aligned}
$$

Recall that, by the construction, the coefficients $a_{i, j}$ satisfy the condition

$$
a_{i, j}=0 \quad \text { if } \zeta_{i}^{(j)}=0 \text {. }
$$

Now we are going to replace the coefficients (31) by some close values $\widetilde{a}_{i, j}, \widetilde{b}_{j}$, respectively, aiming at two targets:

(a) to afford the approximation of $\omega(z)$ by the new function

$$
\widetilde{u}(z):=\sup \left\{\sum_{i=1}^{m} \widetilde{a}_{i, j} \ln \left|z_{i}\right|-\widetilde{b}_{j}: j=1, \ldots, m\right\}
$$

so that

$$
|\omega(z)-\widetilde{u}(z)|<\varepsilon, \quad z \in \overline{D_{1} \backslash D_{0}}
$$

(b) to provide the condition of Lemma 2, namely, that for each $z$ such that $0 \leq \widetilde{u}(z) \leq 1$ the maximum in (33) will be attained for no more than $n$ values of $j$.

To guarantee (a) we must retain the nullity of the new coefficients where the old coefficients vanish (see (32)); indeed, if some of the new coefficients $\widetilde{a}_{i, j}$ were non-zero, while $\zeta_{i, j}=0$, then $\sum_{i=1}^{m} \widetilde{a}_{i, j} \ln \left|\zeta_{i}^{(j)}\right|-\widetilde{b}_{j}=-\infty$ so the closeness to the function $\omega$ would be violated.

To reach both purposes we use the following quite standard algebraic considerations. Given a set $N$ of indices $(i, j)$, we consider the set of all matrices

$$
A=\left(\begin{array}{cccccc}
a_{1,1} & \ldots & a_{i, 1} & \ldots & a_{n, 1} & b_{1} \\
\ldots \ldots & \ldots & \ldots & \ldots & \ldots & \ldots \\
a_{1, j} & \ldots & a_{i, j} & \ldots & a_{n, j} & b_{j} \\
\ldots \ldots \ldots & \ldots \ldots \ldots \ldots \ldots & \ldots & \ldots & \ldots \\
a_{1, m} & \ldots & a_{i, m} & \ldots & a_{n, m} & b_{m}
\end{array}\right)
$$

such that $a_{i, j}=0$ when $(i, j) \in N$. We identify this set of matrices with the space $\mathbb{R}^{d}$, where $d=m(n+1)-\# N$ (writing, for example, the matrix terms row-by-row and dropping those which are the prescribed zeros). Each minor $M$ of a matrix $A$ of order $r$ may then be considered as a homogeneous polynomial $M(A)$ of degree $\leq r$ in $\mathbb{R}^{d}$. Denote by $\mathcal{M}$ the set of all non-trivial minors $M$, i.e. such that $M(A) \not \equiv 0$ on $\mathbb{R}^{d}$. Then the set $\mathcal{A}_{0}$ of all matrices 
$A \in \mathbb{R}^{d}$ such that $M(A) \neq 0$ for all $M \in \mathcal{M}$ is an open dense set in $\mathbb{R}^{d}$, since it can be obtained by removing some algebraic set from $\mathbb{R}^{d}$. It is clear that, by the construction, each matrix $A \in \mathcal{A}_{0}$ satisfies the condition: for every set $J=\left\{j_{1}, \ldots, j_{n+1}\right\}$ with $j_{1}<\ldots<j_{n+1}$ the system

$$
a_{1, j} x_{1}+\ldots+a_{n, j} x_{n}=b_{j}, \quad j \in J,
$$

has no solution. Analogously, we can show that there exists an open dense subset $\mathcal{A}_{1}$ obtained by removing some algebraic set from $\mathbb{R}^{d}$ and such that each matrix $A \in \mathcal{A}_{1}$ satisfies the condition: each system

$$
a_{1, j} x_{1}+\ldots+a_{n, j} x_{n}=1+b_{j}, \quad j \in J
$$

has no solution when $\# J=n+1$. Thus for any matrix $A \in \mathcal{A}_{0} \cap \mathcal{A}_{1}$ each of the systems (36) and (37) has no solution if $\# J=n+1$.

Applying the above considerations to the matrix (35) defined by (31) (with $N$ determined by the condition (32)), we can choose the coefficients $\widetilde{a}_{i, j}$ and $\widetilde{b}_{j}$ to be rational and such that the condition (34) holds and the condition (b) is valid for all $z$ such that $\widetilde{u}(z)=1$ or $\widetilde{u}(z)=0$. Let us show that (b) is also true for all $z$ such that $0<\widetilde{u}(z)<1$. Supposing the contrary, we find that there is $z$ such that $\widetilde{u}(z)=\alpha, 0<\alpha<1$, and

$$
\sum_{i=1}^{n} \widetilde{a}_{i, j} \ln \left|z_{i}\right|=\alpha, \quad j \in J,
$$

for some $J$ with $\# J>n$. Then, by Lemma $3,\left|z_{i}\right|=\left|z_{i}^{(0)}\right|^{1-\alpha} \cdot\left|z_{i}^{(1)}\right|^{\alpha}$ for some $z^{(0)}$ and $z^{(1)}$ such that $\widetilde{u}\left(z^{(0)}\right)=0$ and $\widetilde{u}\left(z^{(1)}\right)=1$. Hence, by what is proved above, there is $j_{0} \in J$ such that $\sum_{i=1}^{n} \widetilde{a}_{i, j_{0}} \ln \left|z_{i}^{(1)}\right|<1$, while $\sum_{i=1}^{n} \widetilde{a}_{i, j_{0}} \ln \left|z_{i}^{(0)}\right| \leq 0$. The last two estimates contradict (38) if $j=j_{0}$. Thus, the condition (b) is proved for all $z$ such that $0 \leq \widetilde{u}(z) \leq 1$.

Since the numbers $\widetilde{a}_{i, j}$ are rational, there exist natural numbers $k_{i}(j)$ and $q$ such that $\widetilde{a}_{i, j}=k_{i}(j) / q, j=1, \ldots, m$. It is easy to check that the numbers $k_{i}(j), q, c_{j}:=q \widetilde{b}_{j}$, and the function $v(z):=\widetilde{u}(z)$ satisfy all the conditions of the lemma. Thus the proof is complete.

Proof of Theorem 4. We shall use the notation

$$
D_{\alpha}:=\left\{z \in D_{1}: \omega(z)<\alpha\right\}, \quad K_{\alpha}:=\left\{z \in D_{1}: \omega(z) \leq \alpha\right\}
$$

with $0<\alpha<1$. Take a sequence $\left(\varepsilon_{\nu}\right)$ such that

$$
5 \varepsilon_{\nu}<\varepsilon_{\nu-1}, \quad \nu=2,3, \ldots, \quad \varepsilon_{1}<1 / 2 .
$$

Now, by Lemma 6 , for each $\nu$ we can find $k(j)=k(j, \nu)=\left(k_{i}(j, \nu)\right) \in \mathbb{Z}_{+}^{n}$, $q=q_{\nu} \in \mathbb{N}$ and real numbers $c_{j}=c(j, \nu), j=1, \ldots, m=m_{\nu}$, such that the estimate (27) holds for the function $v(z)=v_{\nu}(z)$ with $\varepsilon=\varepsilon_{\nu+1}$ and the maximum in (26) is attained for no more than $n$ values of $j$. 
Now we can see that all the conditions of Lemma 2 are fulfilled with $l=n$ for the polynomial polyhedral pair $(L, G)=\left(L^{(\nu)}, G^{(\nu)}\right)$ determined by the normalized equilateral frame $\left[D_{1}, g ; r^{(\nu)}\right]$ with $g=\left(g_{j}\right)=\left(g_{j, \nu}\right): \mathbb{C}^{n} \rightarrow \mathbb{C}^{m_{\nu}}$, where

$$
g_{j, \nu}(z):=\frac{z^{k(j, \nu)}}{\exp \left(2 \varepsilon_{\nu} q_{\nu}-c(j, \nu)\right)}, \quad r^{(\nu)}:=\exp \left(1-4 \varepsilon_{\nu}\right) q_{\nu}
$$

This pair can be expressed in the form

$$
G^{(\nu)}=\left\{z \in D_{1}: v_{\nu}(z)<1-2 \varepsilon_{\nu}\right\}, \quad L^{(\nu)}=\left\{z \in D_{1}: v_{\nu}(z) \leq 2 \varepsilon_{\nu}\right\} .
$$

It is easy to check that the embeddings

$$
D_{1-3 \varepsilon_{\nu}} \Subset G^{(\nu)} \Subset D_{1-\varepsilon_{\nu}}, \quad K_{\varepsilon_{\nu}} \Subset L^{(\nu)} \Subset K_{3 \varepsilon_{\nu}}
$$

hold for all $\nu \in \mathbb{N}$. Thus, applying Lemma 2 to the above polynomial polyhedral pair, for any $\nu \in \mathbb{N}$ we get, by the construction (11), polynomial mappings $\left(g_{i, \nu}^{(s)}\right)_{i=1}^{n}: \mathbb{C}^{n} \rightarrow \mathbb{C}^{n}$ such that the sequence

$$
\varphi_{\nu}^{(s)}(z):=\frac{1}{s n ! \ln r^{(\nu)}} \max \left\{\ln \left|g_{i, \nu}^{(s)}(z)\right|: i=1, \ldots, n\right\}
$$

converges uniformly on $\overline{G^{(\nu)} \backslash L^{(\nu)}}$ to the function

$$
\varphi_{\nu}(z):=\frac{v_{\nu}(z)-2 \varepsilon_{\nu}}{1-2 \varepsilon_{\nu}}
$$

as $s \rightarrow \infty$. Choose $s=s_{\nu}$ so large that

$$
\left|\varphi_{\nu}(z)-\varphi_{\nu}^{\left(s_{\nu}\right)}(z)\right|<\varepsilon_{\nu+1}, \quad z \in \overline{G^{(\nu)} \backslash L^{(\nu)}} .
$$

Hence for the function $u_{\nu}(z):=\varphi_{\nu}^{\left(s_{\nu}\right)}(z)(1-2 \varepsilon)+2 \varepsilon$ we get

$$
\left|u_{\nu}(z)-\omega(z)\right|<2 \varepsilon_{\nu+1}, \quad z \in \overline{G^{(\nu)} \backslash L^{(\nu)}} .
$$

Then the sequence of polyhedral pairs $\left(M^{(\nu)}, H^{(\nu)}\right)$ defined by

$$
\begin{aligned}
M^{(\nu)} & :=\left\{z \in G^{(\nu)}: u_{\nu}(z) \leq 4 \varepsilon_{\nu}\right\}, \\
H^{(\nu)} & :=\left\{z \in G^{(\nu)}: u_{\nu}(z)<1-4 \varepsilon_{\nu}\right\}
\end{aligned}
$$

is as desired. First, it is easy to check that, due to (43) and (40), the embeddings

$$
\begin{aligned}
& G^{(\nu-1)} \Subset D_{1-\varepsilon_{\nu-1}} \Subset H^{(\nu)} \Subset D_{1-3 \varepsilon_{\nu}} \Subset G^{(\nu)}, \\
& L^{(\nu)} \Subset K_{3 \varepsilon_{\nu}} \Subset M^{(\nu)} \Subset K_{\varepsilon_{\nu-1}} \Subset L^{(\nu-1)}
\end{aligned}
$$

hold for $\nu=2,3, \ldots$ So, $H^{(\nu)} \Uparrow D_{1}$ and $M^{(\nu)} \Downarrow \bar{D}_{0}$. Second, these pairs are determined by the normalized equilateral frames (25) with the polynomial 
mapping $f^{(\nu)}=\left(f_{i}^{(\nu)}\right): \mathbb{C}^{n} \rightarrow \mathbb{C}^{n}$ such that

$$
f_{i}^{(\nu)}(z):=\frac{g_{i, \nu}^{\left(s_{\nu}\right)}(z)}{\exp \left(\frac{2 \varepsilon_{\nu}\left(1-4 \varepsilon_{\nu}\right) q_{\nu} s_{\nu} n !}{1-\varepsilon_{\nu}}\right)}, \quad \alpha_{\nu}:=\frac{1-2 \varepsilon_{\nu}}{\left(1-4 \varepsilon_{\nu}\right)\left(1-8 \varepsilon_{\nu}\right) q_{\nu} s_{\nu} n !} .
$$

Finally, by the construction, the formula (24) holds uniformly on any compact subset of $D_{1} \backslash \bar{D}_{0}$.

Proof of Corollary 5. First, we can assume that the set $\Lambda^{(\nu)}:=\{z \in$ $\left.G^{(\nu)}: f^{(\nu)}(z)=0\right\}$ consists only of simple roots (otherwise we can change the polynomial map $f^{(\nu)}$ a little to provide this, preserving all other properties in Theorem 4). Setting $\alpha^{(\nu)}:=\left(\alpha_{\nu}, \ldots, \alpha_{\nu}\right)$, we get

$$
\omega\left(H^{(\nu)}, M^{(\nu)} ; z\right)-1=g_{H^{(\nu)}}\left(\Lambda^{\nu}, \alpha^{(\nu)} ; z\right), \quad z \in H^{(\nu)} \backslash M^{(\nu)} .
$$

Then, since

$$
g_{D_{1}}\left(\Lambda^{(\nu)}, \alpha^{(\nu)} ; z\right) \leq g_{H^{(\nu)}}\left(\Lambda^{(\nu)}, \alpha^{(\nu)} ; z\right) \leq g_{D_{1}}\left(\Lambda^{(\nu)}, \alpha^{(\nu)} ; z\right)+\varepsilon_{\nu-1}
$$

everywhere in $H^{(\nu)}$ and $\omega\left(H^{(\nu)}, M^{(\nu)} ; z\right)$ converges to $\omega(D, K ; z)$ uniformly on compact subsets of $D \backslash K$, we get the relation (4) uniformly on any compact subset of $D \backslash K$.

6. Application to width asymptotics. The Kolmogorov widths of a compact set $A$ in a Banach space $X$ are the numbers

$$
d_{s}(A)=d_{s}(A, X):=\inf _{L} \sup _{x \in A} \inf \left\{\|x-y\|_{X}: y \in L\right\}, \quad s \in \mathbb{Z}_{+},
$$

where $L$ runs through the set of all $s$-dimensional subspaces of $X$.

Let $K$ be a compact subset in an open set $D \subset \mathbb{C}^{n}$ and $A_{K}^{D}$ the subset of the Banach space $C(K)$ consisting of all analytic functions in $D$ whose moduli do not exceed 1 there.

In [23] we conjectured the strong asymptotics

$$
\ln d_{s}\left(A_{K}^{D}\right) \sim-\sigma s^{1 / n}
$$

with the constant

$$
\sigma=\left(\frac{n !}{\tau(K, D)}\right)^{1 / n}
$$

(see (2)). For the one-dimensional case, this conjecture is equivalent to Kolmogorov's conjecture about the asymptotics of the $\varepsilon$-entropy of the compact set $A_{K}^{D}$; see, e.g., [24] for the history of the problem in that case. The conjecture was proved in [1] for Reinhardt pairs $(K, D)$, using the Rauch-Taylor result about the computation of the real Monge-Ampère operator from convex functions.

Now we obtain this fact as a simple consequence of Corollary 5 and the results from $[24$, items 3.1.2, 3.2.5]. Namely it was shown in [24, Proposition 
3.1.4] that under the conditions which are obviously fulfilled in our case, the asymptotics (46) with the constant (47) holds whenever Problem 1 is answered positively for a pair $(K, D)$. So, by Corollary 5 we get

Theorem 7. Let $K=\bar{D}_{0}, D=D_{1}, K \subset D, D_{\nu}$ be bounded logarithmically convex domains, $\nu=1,2$. Then the asymptotics (46) holds with the constant (47).

Notice that recently $([12,10,11])$ a more general result was obtained in a similar way from the complete solution of Problem 1 considered there.

7. Connection with some extremal problem. For any pluriregular pair $(K, D)$ and $m \in \mathbb{N}$ we consider the characteristic

$$
\tau_{m}^{+}(K, D):=\min \left\{\sum_{\mu=1}^{m}\left(\alpha_{\mu}\right)^{n}\right\},
$$

where the minimum is taken over all $\alpha=\left(\alpha_{\mu}\right) \in \mathbb{R}_{+}^{m}$ for which there is $\Lambda=\left(\zeta_{1}, \ldots, \zeta_{m}\right) \in K^{m}$ such that $g_{D}(\Lambda, \alpha ; z) \leq-1, z \in K$. It is easy to show that for every $m \in \mathbb{N}$ there exist $\Lambda=\bar{\Lambda}^{(m)}$ and $\alpha=\bar{\alpha}^{(m)}$ for which the minimum in (48) is attained.

It is obvious that the sequence (48) is non-decreasing, so the limit

$$
\lim _{m \rightarrow \infty} \tau_{m}(K, D)=: \tau^{+}(K, D)
$$

exists. The last characteristic was introduced in [23, 24] and used there to estimate the Kolmogorov widths $d_{s}\left(A_{K}^{D}\right)$ (see the previous section); it was also shown there that a positive solution of Problem 1 for a given pluriregular pair $(K, D)$ leads to the equality

$$
\tau^{+}(K, D)=\tau(K, D)
$$

It follows from [12, Lemma 4.2] that the extremal sequence of pluripotentials $g_{D}\left(\bar{\Lambda}^{(m)}, \bar{\alpha}^{(m)} ; z\right)$ converges to $\omega(D, K ; z)-1$ uniformly on any compact subset of $D \backslash K$. Therefore Problem 2 is equivalent to the following

Problem 3. Is the relation (50) true for any pluriregular pair $(K, D)$ ?

\section{References}

[1] A. Aytuna, A. Rashkovskii, and V. Zahariuta, Width asymptotics for a pair of Reinhardt domains, Ann. Polon. Math. 78 (2002), 31-38.

[2] A. Aytuna and V. Zahariuta, On Lelong-Bremermann Lemma, preprint.

[3] E. Bedford and B. A. Taylor, A new capacity for plurisubharmonic functions, Acta Math. 149 (1982), 1-40.

[4] M. J. Bremermann, On the conjecture of the equivalence of the plurisubharmonic functions and Hartogs functions, Math. Ann. 131 (1956), 76-86. 
[5] R. Gunning, Introduction to Holomorphic Functions of Several Complex Variables, Vol. 1, Wadsworth and Brooks-Cole, Pacific Grove, CA, 1990.

[6] D. Hilbert, Über die Entwicklung einer beliebigen analytischen Funktion einer Variabeln in eine unendliche nach ganzen rationalen Funktionen fortschreitende Reihe, Göttinger Nachr. 1897, 63-70.

[7] L. Hörmander, An Introduction to Complex Analysis in Several Variables, NorthHolland, 1973.

[8] A. N. Kolmogorov and V. M. Tikhomirov, $\varepsilon$-entropy and $\varepsilon$-capacity of sets in function spaces, Uspekhi Mat. Nauk 14 (1959), no. 2, 3-86 (in Russian).

[9] P. Lelong, Notions capacitaires et fonctions de Green pluricomplexes dans les espaces de Banach, C. R. Acad. Sci. Paris Sér. I Math. 305 (1987), 71-76.

[10] S. Nivoche, Proof of the Zakharyuta's conjecture about a Kolmogorov's problem, prépublication no. 213, Laboratoire de mathématiques Emile Picard, Univ. Paul Sabatier, Toulouse III, 2001.

[11] - Sur une conjecture de Zahariuta et un problème de Kolmogorov, C. R. Acad. Sci. Paris Sér. I Math. 333 (2001), 839-843.

[12] E. Poletsky, Negative plurisubharmonic functions. I, preprint, 2001.

[13] T. Ransford, Potential Theory in the Complex Plane, Cambridge Univ. Press, 1995.

[14] J. Rauch and B. A. Taylor, The Dirichlet problem for the multidimensional MongeAmpère equation, Rocky Mountain J. Math. 7 (1977), 345-364.

[15] A. Sadullaev, Plurisubharmonic measures and capacities on complex manifolds, Russian Math. Surveys 36 (1981), 61-119.

[16] J. Siciak, On some extremal plurisubharmonic functions and their applications in the theory of analytic functions of several complex variables, Trans. Amer. Math. Soc. 105 (1962), 322-357.

[17] - Separately analytic functions and envelopes of holomorphy of some lower dimentional subsets of $\mathbb{C}^{n}$, Ann. Polon. Math. 22 (1969), 145-171.

[18] —, Extremal plurisubharmonic functions in $\mathbb{C}^{n}$, ibid. 22 (1981), 175-211.

[19] N. I. Skiba and V. P. Zahariuta, Estimates of $n$-diameters of some classes of functions analytic on Riemann surfaces, Math. Notes 19 (1976), 525-532.

[20] J. L. Walsh, Interpolation and Approximation by Rational Functions, Colloq. Publ. 20, Amer. Math. Soc., Providence, 1960.

[21] V. P. Zahariuta, On extendible bases in spaces of analytic functions of one and several variables, Siberian Math. J. 8 (1967), 204-216.

[22] —, Extremal plurisubharmonic functions, Hilbert scales, and the isomorphism of spaces of analytic functions of several variables, I, II, in: Teor. Funktsiu Funktsional. Anal. i Prilozhen. 19 (1974), 133-157; 21 (1974), 65-83 (in Russian).

[23] —, Spaces of analytic functions and maximal plurisubharmonic functions, D.Sc. thesis, Rostov State Univ., 1984 (in Russian).

[24] - Spaces of analytic functions and complex potential theory, Linear Topol. Spaces Complex Anal. 1 (1994), 74-146.

[25] —, On approximation of pluripotentials by multipolar ones, preprint, 2001.

Sabanci University

81474 Tuzla

Istanbul, Turkey

E-mail: zaha@sabanciuniv.edu.tr
Middle East Technical University Ankara, Turkey 